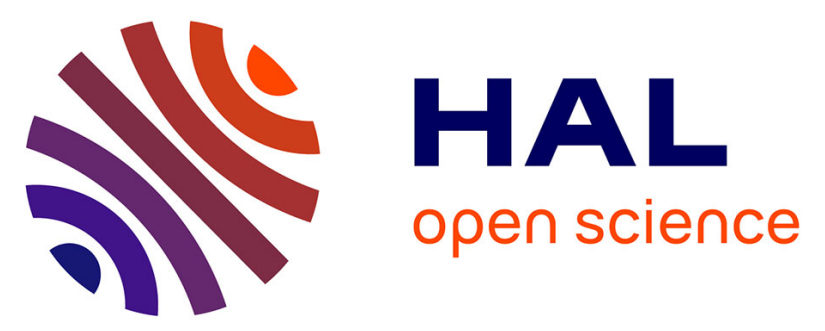

\title{
Some considerations on acoustic resonator phase noise modeling and recent short-term stability experimental results
}

F. Sthal, J. Imbaud, M. Devel, P. Salzenstein, R. Bourquin, Gilles Cibiel

\section{- To cite this version:}

F. Sthal, J. Imbaud, M. Devel, P. Salzenstein, R. Bourquin, et al.. Some considerations on acoustic resonator phase noise modeling and recent short-term stability experimental results. 5th Joint Conference of the 65th IEEE International Frequency Control Symposium/25th European Frequency and Time Forum, May 2011, San Francisco, United States. pp.178-182, 10.1109/FCS.2011.5977810 . hal-00660598

\section{HAL Id: hal-00660598 \\ https://hal.science/hal-00660598}

Submitted on 6 May 2021

HAL is a multi-disciplinary open access archive for the deposit and dissemination of scientific research documents, whether they are published or not. The documents may come from teaching and research institutions in France or abroad, or from public or private research centers.
L'archive ouverte pluridisciplinaire HAL, est destinée au dépôt et à la diffusion de documents scientifiques de niveau recherche, publiés ou non, émanant des établissements d'enseignement et de recherche français ou étrangers, des laboratoires publics ou privés. 


\section{Some considerations on acoustic resonator phase noise modeling and recent short-term stability experimental results}

\author{
F. Sthal ${ }^{\#}$, J. Imbaud ${ }^{\#}$, M. Devel", \\ P. Salzenstein ${ }^{\#}$, R. Bourquin ${ }^{\#}$ \\ \#Frequency and Time Department \\ *Micro-Nano Sciences and Systems Department \\ FEMTO-ST Institute \\ ENSMM, CNRS, UFC, UTBM \\ Besançon, France \\ fsthal@ens2m.fr
}

\author{
G. Cibiel \\ Microwave and Time-Frequency Department \\ CNES (Centre National d'Etudes Spatiales - French National \\ Space Study Center) \\ Toulouse, France
}

\begin{abstract}
This paper presents a critical review of different acoustic resonator models exposed in the past, in the light of recent experimental results. Among the few models attempting to explain the $1 / f$ behavior of the quartz crystal resonator, the $1 / f$ quantum noise theory of $P$. Handel and the thermal boson theory of M. Planat, are recalled. The latest published experimental results are analyzed taking into account these models. In the case of Handel's model, the noise depends on the resonator volume under the electrodes and the unloaded quality factor of the resonator. Handel's model is supposed to give an order of magnitude of the lowest possible noise level, but now some experimental results are under its prediction. An approach using the vibrating volume of the resonator is proposed to renew the lowest noise level estimation and found to be compatible with the latest experimental results.
\end{abstract}

\section{INTRODUCTION}

High-precision quartz oscillators are mainly used in telecommunications, metrology and space applications such as local oscillators in satellites. The short-term stability of such oscillators is essential.

In 1994, J. Norton [1] has measured the best result of short-term stability of a quartz crystal resonator. It was a $5 \mathrm{MHz}$ SC cut mounted in a BVA structure. The result in term of Allan standard deviation was $3.74 \cdot 10^{-14}$ for $\tau$ equals to $10 \mathrm{~s}$. In 1999, at $10 \mathrm{MHz}$, R.J. Besson [2] measured two BVA quartz crystal oscillators with $5 \cdot 10^{-14}$ Allan standard deviation. Then, for ten years, there had been no significant improvement of quartz oscillators in the short term stability domain and no real solution had been found to improve the phase noise of BAW resonators. J. Norton's result represented the best acoustic resonator noise performance that could be found in the literature, pretty close to the lowest theoretical estimates. However, recently, better experimental results [3-4] have shown that quartz crystal oscillators may still have not said their last word in the short-term stability domain.
The oscillator limitation is theoretically imposed by the resonator. However, few models attempt to explain the $1 / f$ behavior of the quartz crystal resonator. In this paper, we will briefly recall the $1 / f$ quantum noise theory of $\mathrm{P}$. Handel and the thermal boson theory of M. Planat in order to compare the latest published experimental results to the lower limitations computed with these two models. In the case of Handel's model, the noise depends on the resonator volume under the electrodes and the unloaded quality factor $(\mathrm{Q})$ of the resonator. It gives an order of magnitude of the noise, but now some experimental results are below its prediction. In this paper, we will show that a lower theoretical estimate can nonetheless be extracted from Handel's model provided one uses the vibrating volume of the resonator instead of the volume under the electrodes.

\section{MODELS}

The following part presents a summary of the two models trying to elucidate the physical origin of the $1 / f$ flicker noise of a quartz crystal resonator with a focus on the origin of the $1 / f$ formula. We note, that both models can be seen as the two opposite sides of the fluctuation-dissipation theorem [5], since Planat studies the energy fluctuations around statistical equilibrium of a population of massless degenerate thermal bosons, whereas Handel proposes an origin for the dissipation occurring when the oscillator comes back to its mode after a small perturbation.

\section{A. Planat's Model}

In 2003, M. Planat proposed a statistical thermodynamics based model to explain the $1 / f$ noise in quartz crystal resonators [6]. In this paper, Planat considered the case of a population of massless bosons with a degenerate quantum harmonic oscillator energy spectrum which predict a hyperbolic fluctuation spectrum at low frequencies. According to the equipartition law of statistical mechanics, the partition function is introduced using a degeneracy parameter of the 
energy level $n h f$ with $n$ the number of bosons, $h$ the Planck constant and $f$ the frequency. The free energy $\mathrm{F}$ may be approximated in the low frequency part of the spectrum by:

$$
\frac{F}{k T} \approx-\frac{\pi^{2}}{6 x}-\frac{1}{2} \ln \left(\frac{x}{2 \pi}\right)+\frac{x}{24}
$$

with $x=h f / k T$ of (1).

Subsequently, the exposed model keeps only the first term

According to the Einstein's conventional approach, the energy fluctuations of a system in equilibrium within a larger system of temperature $T$ are:

$$
\varepsilon^{2}=\left\langle(E-\langle E\rangle)^{2}\right\rangle=k T^{2} \frac{\partial\langle E\rangle}{\partial T}
$$

One can reformulate this relation for the fluctuations of the energy $\mathrm{dE}=\mathrm{u}(f, \mathrm{~T}) \mathrm{d} f$ in the bandwidth $\mathrm{d} f$ :

$$
d \varepsilon^{2}=k T^{2} \frac{\partial u}{\partial T} d f=S_{u}(f) d f
$$

with $u$ the energy spectral density and $\mathrm{S}_{\mathrm{u}}(f)$ the power spectral density of the fluctuations of $u$.

Thus, energy fluctuations in the bandwidth $\mathrm{d} f$ are given by:

$$
\left[\frac{S_{u}(f)}{u^{2}}\right]_{L F}=\frac{9 h c_{p h}^{3}}{4 \pi^{3} V} \frac{1}{k T f}=\frac{A_{p h}}{V f}
$$

With $3 / c_{p h}^{3}=1 / c_{C}^{3}+1 / c_{B}^{3}+1 / c_{A}^{3}, c_{A}, c_{B}$ and $c_{C}$ the velocities of the longitudinal mode $\mathrm{A}$ and the shear modes $\mathrm{B}$ and $\mathrm{C}$, respectively.

Considering [7-8], the fractional frequency fluctuations in a quartz crystal resonator are:

$$
S_{y}(f)=\frac{S_{\omega}(f)}{\omega^{2}}=\frac{1}{Q^{4}} \frac{A_{p h}}{V f}=\frac{h_{-1}}{f}
$$

(5) has been used to give an order of magnitude of the power spectral density of the fractional frequency fluctuations of a $5 \mathrm{MHz} 5^{\text {th }}$ overtone (OT 5), AT-cut quartz crystal resonator.

\section{B. Handel's Model}

P. H. Handel proposes in [9] a summary of his $1 / f$ quantum noise theory. According to him, the general origin of the intrinsic $1 / f$ noise is due to a quantum self-interference process caused by very small spontaneous bremsstrahlung energy losses. The rate $\Gamma$ of phonon interactions which remove phonons from the main quartz resonator mode is modulated by the quantum $1 / f$ effect. The time-derivative of the polarization vector $\mathrm{P}$ of the quartz crystal is affected. According to bremsstrahlung, when the phonon is removed, a constant energy is radiated away per unit frequency interval at any frequency, its expression is:

$$
E=\frac{1}{\left(4 \pi \varepsilon_{0}\right)} \frac{(\Delta \dot{P})^{2}}{3 c^{3}}
$$

With $\Delta \dot{\mathrm{P}}$, the variation of current density of the quartz crystal.

Then the number of photon emitted as bremsstrahlung of frequency $f$, per Hertz is given by dividing (6) by the energy of photon $h f$ :

$$
Z=\frac{2 \alpha(\Delta \dot{P})^{2}}{3 \pi f e^{2} c^{2}}
$$

With

$$
\alpha=\left(1 / 4 \pi \varepsilon_{0}\right)\left(2 \pi e^{2} / h c\right)=1 / 137 \text {, the }
$$

Sommerfeld's fine structure constant.

Thus the one side spectrum is inversely proportional to $f$ :

$$
S_{\delta \Gamma / \Gamma}(f)=\frac{4 \alpha(\Delta \dot{P})^{2}}{3 \pi f e^{2} c^{2}}
$$

If the main resonant mode is described as a forced harmonic oscillator with losses then the quantum $1 / f$ fluctuations are present in the loss coefficient $\Gamma$ of the following equation:

$$
\frac{d^{2} x}{d t^{2}}+2 \Gamma \frac{d x}{d t}+\omega_{0}^{2} x=F(t)
$$

The quality factor of the resonator is defined as:

$$
Q=\omega_{r} / 2 \Gamma
$$

The resonant frequency is given by:

$$
\omega_{r}^{2}=\omega_{0}^{2}-2 \Gamma^{2}
$$

Thus

$$
S_{y}(f)=\frac{1}{4 Q^{4}} S_{\delta \Gamma / \Gamma}(f)
$$

Where $y=\Delta f / f_{0}$ is the relative frequency fluctuation.

The vibrational energy of the crystal can be written as:

$$
W=n \hbar\langle\omega\rangle=2\left(\frac{N m}{2}\right)\left(\frac{d x}{d t}\right)^{2}=\left(\frac{m}{N e^{2}}\right) \chi^{2}(\dot{P})^{2}
$$

With $m$ the reduced mass of the elementary oscillating dipoles, $e$ their charge, $N$ their number in the crystal, $\chi$ a polarization constant, $\langle\omega\rangle$ the average circular frequency of a thermal phonon interacting with phonons in the main resonator mode and $n$ the average number of phonons in that typical thermal phonon mode.

Thus with:

$$
(\Delta \dot{P})^{2}=\frac{N \hbar\langle\omega\rangle}{n} \frac{e^{2}}{4 \chi^{2}}
$$

We can write:

$$
S_{y}(f)=\frac{\beta}{f}\left(\frac{V}{Q^{4}}\right)
$$


With: $\beta=n_{0} \frac{\alpha \hbar\langle\omega\rangle}{12 n \pi m c^{2} \chi^{2}}=1 / \mathrm{cm}^{3}, \mathrm{n}_{0}$ the number of dipoles per volume unit and $V$ in $\mathrm{cm}^{3}$.

\section{ACOUSTIC VOLUME ESTIMATION}

The acoustic volume estimation is carried out using Tiersten's definitions as $e . g$. in [10].

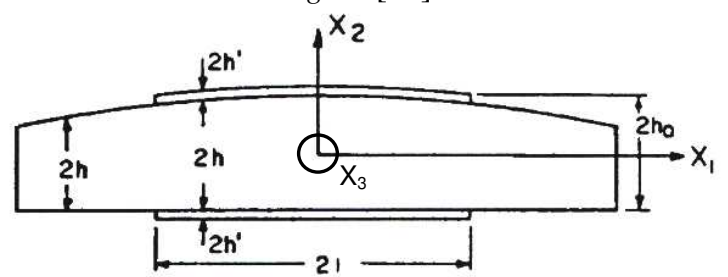

Figure 1. Geomeric definition of a plano-convex resonator

Due to the plano-convex shape, the thickness of the resonator $2 h$ is given by the following expression (Fig. 1):

$$
2 h \cong 2 h_{0}\left[1-\frac{\left(x_{1}^{2}+x_{3}^{2}\right)}{4 R h_{0}}\right]
$$

The amplitude of the mechanical displacement in the resonator is:

$$
\begin{aligned}
u_{n m p}= & A \sin \frac{n \pi x_{2}}{2 h} H_{m}\left(\sqrt{\alpha_{n}} x_{1}\right) H_{p}\left(\sqrt{\beta_{n}} x_{3}\right) \\
& \cdot e^{-\alpha_{n}\left(x_{1}^{2} / 2\right)} e^{-\beta_{n}\left(x_{3}^{2} / 2\right)} e^{j \omega_{n m p} t}
\end{aligned}
$$

with:

$$
\alpha_{n}^{2}=\frac{n^{2} \pi^{2} \hat{\mathrm{c}}}{8 R h_{0}^{3} M_{\mathrm{n}}} \text { and } \beta_{n}^{2}=\frac{n^{2} \pi^{2} \hat{\mathrm{c}}}{8 R h_{0}^{3} P_{\mathrm{n}}}
$$

Where $n=1,3,5, \ldots$ the overtone number, $m, p=0,2,4$, ..., label the different mode shapes in the plane of the resonator, $H_{m}$ and $H_{p}$ are Hermit polynomials, $R$ is the curvature radius, $2 h_{0}$ is the thickness of the resonator center, $M_{n}$ and $P_{n}$ are the dispersion constants and $\hat{c}$ is the effective elastic constant associated to the propagation of the (C) mode.

For the $3^{\text {rd }}$ overtone (OT 3), we have:

$$
\begin{gathered}
u_{300}=A \sin \frac{3 \pi x_{2}}{2 h} e^{-\alpha_{3}\left(x_{l}^{2} / 2\right)} e^{-\beta_{3}\left(x_{3}^{2} / 2\right)} e^{j \omega_{300} t} \\
\alpha_{3}^{2}=\frac{9 \pi^{2} \hat{\mathrm{c}}}{8 R h_{0}^{3} M_{3}} \text { and } \beta_{3}^{2}=\frac{9 \pi^{2} \hat{\mathrm{c}}}{8 R h_{0}^{3} P_{3}}
\end{gathered}
$$

In the case of SC-cut quartz resonator [11], the cut is defined by a doubly rotation $\left(\varphi=22^{\circ} 45^{\prime}, \theta=34^{\circ}\right)$ and in $\mathrm{N} / \mathrm{m}^{2}$ :

$$
\hat{c} \cong 34.6 \cdot 10^{9}, M_{3} \cong 57 \cdot 10^{9}, P_{3} \cong 67 \cdot 10^{9}
$$

The energy of the acoustic wave is given by:
$W_{1} \cong \frac{1}{2} \hat{c}\left(\frac{3 \pi}{2 h}\right)^{2} \iiint_{V}\left[\cos \left(\frac{3 \pi}{2 h} x_{2}\right) e^{-\alpha_{3} \frac{x_{1}^{2}}{2}} e^{-\beta_{3} \frac{x_{3}^{2}}{2}}\right]^{2} d V$

To define the acoustic volume, we can consider the vibration amplitude which remains constant over the surface $\mathrm{S}_{\mathrm{eq}}$, then we compute a cylindrical volume that has the same energy than the acoustic wave.

$$
W_{2} \cong \frac{1}{2} \hat{c}\left(\frac{3 \pi}{2 h}\right)^{2} \int\left[\cos \left(\frac{n \pi}{2 h} x_{2}\right) d x_{2}\right]^{2} \times S_{e q}
$$

Thus:

$$
W_{l}=W_{2} \Rightarrow S_{e q}=\iint_{S}\left[e^{-\alpha_{3} \frac{x_{1}^{2}}{2}} e^{-\beta_{3} \frac{x_{3}^{2}}{2}}\right]^{2} d x_{1} d x_{3}
$$

Consequently, the acoustic volume is defined by:

$$
V_{a c}=S_{e q} 2 h_{0}
$$

\section{APPLICATION OF THE MODELS TO EXPERIMENTAL MEASUREMENTS}

As seen in [4], the best measured resonators are the $5 \mathrm{MHz}$ BVA SC-cut, OT 3, resonator from Oscilloquartz (Neuchatel, Switzerland). In [12], a succinct description of the resonator is given which allows us to compute an order of magnitude of our approach. The diameter of the resonant part of the resonator is around $20 \mathrm{~mm}$, the electrodes diameter $D$ is about $11 \mathrm{~mm}$. The thickness of the resonator is chosen to vibrate on the OT 3 at $5 \mathrm{MHz}$. The acoustic velocity for the thickness shear C-mode of a SC-cut in quartz crystal is about $3610 \mathrm{~m} / \mathrm{s}$. Thus, the thickness at the center of the resonator $2 h_{0}$ is given by $3 \lambda / 2$ formula and is approximately equal to $1.15 \mathrm{~mm}$.

The energy trapping is quasi-independent of the electrodes dimension, in contoured resonators. It influences the motional resistance and the static capacitor. The displacement can be computed from the center of the resonator to the external edge of the resonator. The radius of curvature is one of the most important parameter. Usually, sufficient energy trapping is obtained when the lateral boundary has no effect on the vibration. Thus, we will consider an attenuation of the mechanical displacement of about $10^{-7}$ between the center and the boundary of the resonator. This trapping corresponds to a curvature radius $R$ of about $290 \mathrm{~mm}$ (fig. 2).

The $\mathrm{Q}$ factor of this kind of resonator can be found from the dissipative matrix $\eta_{i j}$ of the quartz crystal [13]. These constants must be expressed in the SC-cut orientation. Then, the values are considered as the imaginary part of the effective elastic coefficients. Resolution of the Christoffel equations [11] gives the complex eigenvalue $\hat{c}+i \hat{\eta}$. Then, an order of magnitude of the $\mathrm{Q}$ factor can be obtained using the following equation: 


$$
Q=\frac{\hat{c}}{\hat{\eta} 2 \pi f}
$$

In our case, $\hat{\eta} \cong 3.95 \cdot 10^{-4} \mathrm{Ns} / \mathrm{m}^{2}$. A SC-cut quartz crystal resonator, at $5 \mathrm{MHz}$, OT 3 , gives a maximum of quality factor of about $2.8 \cdot 10^{6}$. This value corresponds with the one given in [3].

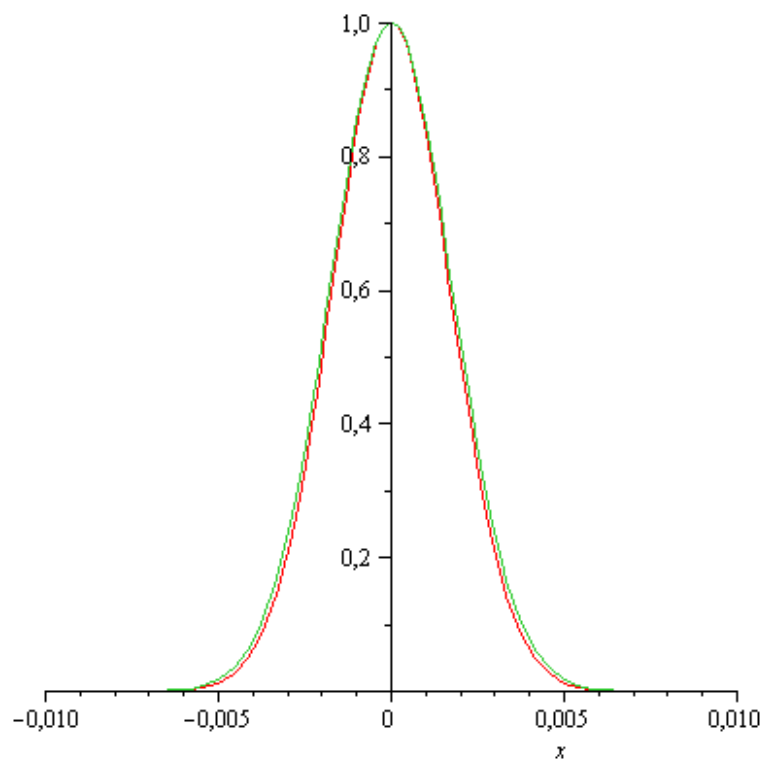

Figure 2. Energy trapping of a plano-convex resonator, $\mathrm{SC}$-cut $5 \mathrm{MHz}$, OT3, $R=290 \mathrm{~mm}$. (red curve: along $\mathrm{x}_{3}$ axis, green curve: along $\mathrm{x}_{1}$ axis).

Consequently, we can compute the motional resistor $\mathrm{R}_{\mathrm{mot}}$ from the motional capacitance $\mathrm{C}_{\mathrm{mot}}$ using:

$$
R_{m o t}=\frac{1}{Q C_{m o t} 2 \pi f}=\frac{L_{m o t} 2 \pi f}{Q}
$$

In the case of OT $3, \mathrm{C}_{\mathrm{mot}}$ is given by [10]:

$$
C_{m o t}=\frac{64 \hat{e}_{26}^{2}\left(\int_{0}^{\frac{D}{2}} e^{-\alpha_{3} \frac{x_{1}^{2}}{2}} d x_{1} \int_{0}^{\frac{D}{2}} e^{-\beta_{3} \frac{x_{3}^{2}}{2}} d x_{3}\right)^{2} \sqrt{\alpha_{3}} \sqrt{\beta_{3}}}{9 \pi^{3} h_{0} \hat{c}}
$$

With $D$ the electrode diameter, $\hat{e}_{26} \cong-0.0576 \mathrm{C} / \mathrm{m}^{2}$ the effective piezoelectric constant.

The computing value of $C_{m o t}$ is around $280 \mathrm{fF}$. We obtain $R_{\text {mot }}$ equals to $42 \Omega, L_{\text {mot }}$ is about $3.6 \mathrm{H}$. These results are closed to the measured value in [3].

The static capacitor $C_{0}$ can be also computed with the following formula [9]:

$$
C_{0}=\frac{\pi D^{2}}{4}\left(1+\frac{(D / 2)^{2}}{8 R h_{0}}\right) \frac{\varepsilon_{22}+\frac{\hat{e}_{26}^{2}}{\hat{c}}}{2 h_{0}}
$$

With $\varepsilon_{22}=39.78 \cdot 10^{-12} \mathrm{~F} / \mathrm{m}$ the permittivity constant in SCcut.

We have $C_{0}$ around $3.4 \mathrm{pF}$.

Short-term stability is usually given in the time domain with an Allan variance. The Allan variance is computed from the Fourier frequency and from the power spectral density of the frequency fluctuations [14]:

$$
\sigma_{y}^{2}(\tau)=\int_{0}^{\infty} S_{y}(f) \frac{2 \sin ^{4} \pi f \tau}{(\pi f \tau)^{2}} d f
$$

In the case of the flicker frequency noise (which is the resonator noise), relation between the Allan standard deviation and the power spectral density (PSD) of relative frequency fluctuations $S_{y}(f)$, is given by [15]:

$$
\sigma_{y_{-} \text {floor }}=\sqrt{2 \ln (2) S_{y}(1 \mathrm{~Hz})}
$$

Table 1 presents the comparison between measurements and the results proposed by both models. For Planat's model, only the velocity of the C-mode of the acoustic wave is used to the computation. The result is really higher than the

\begin{tabular}{|c|c|}
\hline Frequency (MHz) & 5 \\
\hline Quality factor $Q\left(10^{6}\right)$ & 2.8 \\
\hline $\mathbf{R}_{\text {mot }}(\Omega)$ & 42 \\
\hline $\mathrm{C}_{\text {mot }}(\mathrm{fF})$ & 280 \\
\hline $\mathbf{L}_{\text {mot }}(\mathbf{H})$ & 3.6 \\
\hline $\mathrm{C}_{0}(\mathrm{pF})$ & 3.4 \\
\hline Volume under electrodes $V_{\text {elec }}\left(\mathrm{cm}^{3}\right)$ & $1.093 \cdot 10^{-1}$ \\
\hline Acoustic volume $V_{\text {ac }}\left(\mathrm{cm}^{3}\right)$ & $1.076 \cdot 10^{-2}$ \\
\hline Volume ratio & 10 \\
\hline $\begin{array}{c}\sigma_{y_{\text {_flloor_planat }}} \\
\text { (vol. under electrodes) }\end{array}$ & $5.2 \cdot 10^{-12}$ \\
\hline $\begin{array}{c}\sigma_{\mathbf{y}_{\_} f l o o r} \text { _Handel } \\
\text { (vol. under electrodes) }\end{array}$ & $5.4 \cdot 10^{-14}$ \\
\hline $\begin{array}{l}\sigma_{\mathbf{y}_{\_} \text {floor_Handel }} \\
\text { (Acoustic vol.) }\end{array}$ & $1.67 \cdot 10^{-14}$ \\
\hline $\begin{array}{c}\sigma_{\mathbf{y}_{\text {ffloor_exp }}[1]} \\
\text { (oscillator measurement) }\end{array}$ & $3.75 \cdot 10^{-14}$ \\
\hline $\begin{array}{c}\sigma_{y_{-} \text {floor_exp }[4]} \\
\text { (oscillator measurement) }\end{array}$ & $2.5 \cdot 10^{-14}$ \\
\hline
\end{tabular}
experiment. Using the acoustic volume in this model is not relevant because of the invert proportionality.

TABLE I. COMPARISON OF THE SHORT-TERM STABILITY IN TERM OF ALLAN STANDARD DEVIATION $\sigma_{\mathrm{Y} \_ \text {FLOR }}$ OF A SC-CUT, $5 \mathrm{MHZ}$, OT 3 RESONATOR

The flicker noise seems to be the limit of the short-term stability of a quartz crystal resonator. An order of magnitude of this limit is given by the previous calculus around $1.610^{-14}$ with the Handel's model using the definition of the acoustic volume. 
If it was possible to eliminate this noise, the noise limit should be given by the thermal noise of the resonator. In term of Allan standard deviation, its expression is usually [16]:

$$
\sigma_{y_{-} \text {therm }}(\tau)=\frac{1}{Q_{L}} \sqrt{\frac{k T}{2 P \tau}}
$$

With a loaded $\mathrm{Q}$-factor $\mathrm{Q}_{\mathrm{L}}$ around $1.6 \cdot 10^{6}$ for the previous resonator and a excitation power of $60 \mu \mathrm{W}$, we get around $\mathrm{T}=80^{\circ} \mathrm{C}$ :

$$
\sigma_{y_{-} \text {therm }}(\tau) \approx 4 \cdot 10^{-15} \cdot \tau^{-1 / 2}
$$

At $1 \mathrm{~s}$, without $1 / f$ noise, the short-term stability limit should be $4 \cdot 10^{-15}$.

\section{CONCLUSION}

Regarding the experimental results, the $1 / f$ story of the quartz crystal is not over. The considered models should be the fundamental limit of the flicker noise of a resonator. Values of Handel's model are under the experimental measurements if the volume is considered as the acoustic volume. The experimental results seem to be now very close to the fundamental limit. The model validity could be proved by varying the acoustic volume while keeping the $\mathrm{Q}$ factor of the resonator constant.

\section{ACKNOWLEDGMENT}

The authors thank M. Planat for his helpful discussion and the CNES for financial support of the work.

\section{REFERENCES}

[1] J. R. Norton, "Performance of ultrastable quartz oscillators using BVA resonators," Proc. European Frequency and Time Forum, Weihenstephan, Germany, March 1994, pp. 457-465.

[2] R. J. Besson, M. Mourey, S. Galliou, F. Marionnet, F. Gonzalez, P. Guillemot, R. Tjoelker, W. Diener, A. Kirk, "10 MHz Hyperstable quartz oscillators," Proc. Joint Meeting IEEE Ann. Freq. Cont. Symp. and European Frequency and Time Forum, Besançon, France, april 2009, pp. 326-330.
[3] J. Chauvin, P. Weber, J.P. Aubry, F. Lefebvre, F. Sthal, S. Galliou, E. Rubiola and X. Vacheret, "A new generation of very high stability BVA oscillators," Proc. Joint Meeting IEEE Ann. Freq. Cont. Symp. and European Frequency and Time Forum, Genova, Switzerland, June 2007, pp. 1261-1268.

[4] P. Salzenstein, A. Kuna, L. Sojdr and J. Chauvin, "Significant step in ultra high stability quartz crystal oscillators," Electron. Lett., vol. 46, no. 21, pp. 1433-1434, (2010).

[5] R. Kubo, "The fluctuation-dissipation theorem," Rep. Prog. Phys., vol. 29, pp. 255-284, (1966).

[6] M. Planat, "Thermal $1 / f$ noise from the theory of partitions: application to a quartz resontor, " Physica A, vol. 318, pp. 371-386, (2003).

[7] J. J. Gagnepain, J. Uebersfeld, G. Goujon, P. Handel, "Relation between 1/f noise and Q-factor in quartz resontors," Proc. IEEE Ann. Freq. Cont. Symp., Ft. Monmouth, New Jersey, May 1981, pp. 476483.

[8] P. H. Handel, "Nature of $1 / f$ frequency fluctuations in quartz resonators," Solid State Electron., vol. 22, pp. 875-876, (1979).

[9] P. H. Handel, "Quantum $1 / f$ effect in resonant biochemical piezoelectric and MEMS sensors," IEEE Trans. on Ultrason., Ferroelec. and Freq. Cont., vol. 52, no. 9, Sept., pp. 1461-1467, (2005).

[10] D. S. Stevens, H. F. Tiersten, "An analysis of doubly rotated quartz resonators utilizing essentially thickness modes with transverse variation," J. Acous. Soc. Am., vol. 79, n 6, pp. 1811-1826, (1986).

[11] R. Bourquin, B. Dulmet, J. J. Boy, "SC-cut resonator operating in anharmonic modes with B-mode reduction," Proc. European Frequency and Time Forum, Brighton, United Kingdom, March 1996, pp. 239243.

[12] D. Schneuwly, Y. Schwab, "Quartz clocks," $3^{\text {rd }}$ Int. Telec. Sync. Forum, London, UK, Oct. 2005.

[13] J. Lamb, J. Richter, "Anisotropic acoustic attenuation with new measurements for quartz at room temperatures," Proc., Roy. Soc. Series A, vol. 293, pp. 479-492, (1966).

[14] L.S. Cutler, C. L. Searle, "Some aspects of the theory and measurment of frequency fluctuations in frequency standards," Proc. IEEE, vol. 54, no. 2, Feb., pp. 136-154, (1966).

[15] F. Sthal, M. Mourey, F. Marionnet, W. F. Walls, "Phase noise measurements of $10 \mathrm{MHz}$ BVA quartz crystal resonators," IEEE Trans. on Ultrason., Ferroelec. and Freq. Cont., vol. 47, no. 2, March, pp. 369373, (2000).

[16] F. L. Walls, J. Vig, Phase Noise "Fundamental limits on the frequency stabilities of crystal oscillators," IEEE Trans. on Ultrason., Ferroelec. and Freq. Cont., vol. 42, no. 4, July, pp. 576-588, (1995). 